\title{
On the Generalized Maxwell-Bloch Equations
}

\author{
Pavle SAKSIDA
}

Department of Mathematics, Faculty of Mathematics and Physics, University of Ljubljana, Slovenia

E-mail: Pavle.Saksida@fmf.uni-lj.si

Received December 01, 2005, in final form March 05, 2006; Published online March 27, 2006

Original article is available at http://www.emis.de/journals/SIGMA/2006/Paper038/

\begin{abstract}
A new Hamiltonian structure of the Maxwell-Bloch equations is described. In this setting the Maxwell-Bloch equations appear as a member of a family of generalized Maxwell-Bloch systems. The family is parameterized by compact semi-simple Lie groups, the original Maxwell-Bloch system being the member corresponding to $S U(2)$. The Hamiltonian structure is then used in the construction of a new family of symmetries and the associated conserved quantities of the Maxwell-Bloch equations.
\end{abstract}

Key words: Maxwell-Bloch equations; Hamiltonian structures; symmetries; conserved quantities

2000 Mathematics Subject Classification: 37K05; 35Q60; 37K30; 35Q58; 53D20

\section{Introduction}

Maxwell-Bloch equations are a system of partial differential equations which plays a prominent role in the field of non-linear optics. This system models the resonant interaction between light and an optically active medium consisting of two-level atoms. The quantities figuring in this system are the complex valued functions $E(t, x), P(t, x): \mathbb{R}^{2} \rightarrow \mathbb{C}$ and a real valued function $D(t, x): \mathbb{R}^{2} \rightarrow \mathbb{R}$ of two independent variables, time $t$ and one spatial variable $x$. The function $E(t, x)$ is the slowly varying envelope of the electric field, $P(t, x)$ is the polarization of the medium and $D(t, x)$ is the population inversion. Here we will consider the MaxwellBloch equations without pumping and in the sharp-line limit, that is, without inhomogeneous broadening:

$$
E_{t}+c E_{x}=P, \quad P_{t}=E D-\beta P, \quad D_{t}=-\frac{1}{2}(\bar{E} P+E \bar{P}) .
$$

The constant $c$ above is the speed of light in the medium and $\beta$ represents the longitudinal relaxation time of the medium. The transverse relaxation time and the losses of the electric field are assumed to be equal to zero.

We will show that the Maxwell-Bloch equations describe a continuous chain of interacting C. Neumann oscillators on the three-sphere. The interactions between the neighbouring oscillators are of magnetic type, which means that the acceleration of any given oscillator depends on the velocity of its neighbours and not on their position. More explicitly, we will show that the system (1) is equivalent (modulo a certain constraint) to the second-order partial differential equation

$$
\left(g_{t} g^{-1}\right)_{t}+c\left(g_{t} g^{-1}\right)_{x}=\left[\sigma, \operatorname{Ad}_{g}(\tau(x))\right]
$$

where $g(t, x): \mathbb{R} \times \mathbb{R} \rightarrow S U(2)$ is a Lie group valued function of two variables, $\sigma \in \mathfrak{s u}(2)$ is a constant and $\tau(x): \mathbb{R} \rightarrow \mathfrak{s u}(2)$ an arbitrary path in the Lie algebra. We shall see that this equation is the equation of motion of the above-mentioned chain of C. Neumann oscillators. 
If we replace the group $S U(2)$ by an arbitrary Lie group $G$, the equation (2) still makes sense. We shall call the equation (2), with $g(t, x): \mathbb{R} \times \mathbb{R} \rightarrow G$ and $\sigma, \tau(x) \in \mathfrak{g}=\operatorname{Lie}(G)$, the generalized Maxwell-Bloch equation. We will show that in the case when $G$ is a compact semi-simple Lie group, the equation (2) is again the equation of motion for a continuous chain of oscillators. In this case, the configuration space of the relevant model oscillator will be the Lie group $G$. These oscillators belong to a class of well-known integrable systems described by Reyman and Semenov-Tian-Shansky in [1] and [2], and later in different contexts by other authors, see e.g. [3].

The rewriting (2) of the Maxwell-Bloch equations proves to be useful in several ways. In this paper, we shall concentrate on two features. First we shall construct and describe a new Hamiltonian structure for the Maxwell-Bloch equations and their generalizations. This will then enable us to find an infinite-dimensional group of symmetries and corresponding infinitedimensional class of conserved quantities of our equation.

We shall limit ourselves to the spatially periodic case

$$
g(t, x+2 \pi)=g(t, x), \quad \tau(x+2 \pi)=\tau(x),
$$

and we shall assume that the group $G$ is compact and semi-simple. We will show that $(2)$ is the equation of motion for the Hamiltonian system $\left(T^{*} L G, \omega_{c}+c \omega_{m}, H_{m b}\right)$. Here the configuration space $L G=\left\{g(x): S^{1} \rightarrow G\right\}$ is the loop group over $G$. The Hamiltonian $H_{m b}: T^{*} L G \rightarrow \mathbb{R}$ is given by the formula

$$
H_{m b}\left(g, p_{g}\right)=\int_{S^{1}}\left(\frac{1}{2}\left\|p_{g}(x)\right\|^{2}+K\left(\sigma, \operatorname{Ad}_{g(x)} \tau(x)\right)\right) \mathrm{d} x
$$

where $K(-,-)$ is the Killing form on $\mathfrak{g}$. The symplectic structure $\omega_{c}+c \omega_{m}$ is the canonical structure $\omega_{c}$ on the cotangent bundle $T^{*} G$, perturbed by the so called magnetic term $c \omega_{m}$. The term $\omega_{m}$ is the pull-back $\pi^{*}\left(\widetilde{\omega}_{m}\right)$, via the natural projection $\pi: T^{*} L G \rightarrow L G$, of a right-invariant differential 2 -form $\widetilde{\omega}_{m}$ on $L G$. The value of $\widetilde{\omega}_{m}$ at the identity is given by

$$
\left(\widetilde{\omega}_{m}\right)_{e}(\xi, \eta)=\int_{S^{1}} K\left(\xi^{\prime}(x), \eta(x)\right) \mathrm{d} x, \quad \xi(x), \eta(x) \in L \mathfrak{g}=T_{e} L G(2) .
$$

We stress that in the case when $G=S U(2)$, the above system provides a new Hamiltonian structure for the Maxwell-Bloch equations (1).

We see that the symplectic structure of our Hamiltonian system is not canonical. It is wellknown that the perturbations of the canonical symplectic forms are responsible for the forces of the magnetic type, see e.g. $[4,5,6]$. The model example of such a situation is the motion of an electrically charged particle in a magnetic field. The Lorentz force can be encoded as a perturbation of the canonical symplectic form on $T^{*} \mathbb{R}^{3}$. One can geometrize the Lorentz-type force by adding another (circular) degree of freedom to the configuration space. On the suitably extended phase space $T^{*} M$ the symplectic form will be canonical. One can then easily find such a metric on $M$ that the geodesics on $M$ will project down to the trajectories of a particle in $N$ under the influence of our magnetic-type force. This procedure is known by the name of the Kaluza-Klein theory. Whenever the magnetic perturbation $\omega_{m}$ is exact, the KaluzaKlein extended space is simply $M=N \times U(1)$. If $\omega_{m}$ is not exact, then the extended space exists under the condition that $\omega_{m}$ has a certain integrality property. In this case, the extended space is a non-trivial $U(1)$-bundle whose Chern class is equal to the de Rham class of $\omega_{m}$. In the case of the generalized Maxwell-Bloch equations the form $\widetilde{\omega}_{m} \in \Omega^{2} L G$ is not exact. The extended configuration space is therefore a non-trivial $U(1)$-principal bundle over $L G$. It is actually precisely the central extension $\widetilde{L} G$ of the loop group $L G$. A detailed exposition of the Kaluza-Klein description of the Maxwell-Bloch equations can be found in [7]. The geometric prerequisites, needed for the construction of non-trivial extended spaces, are given in [8]. Here, 
we would only like to mention the following interesting fact: In the Kaluza-Klein description of the electron moving in a magnetic field, the moment, conjugate to the additional circular degree of freedom, is the charge of the electron. Therefore, also in other situations, this moment is called charge. It is interesting to note that in the case of the Maxwell-Bloch equations the charge is precisely the speed of light in the medium.

As we already mentioned, the important merit of the equation (2) and the corresponding Hamiltonian system $\left(T^{*} L G, \omega_{c}+c \omega_{m}, H_{m b}\right)$ is the fact that they enable us to find an infinitedimensional family of symmetries and the corresponding conserved quantities of the (generalized) Maxwell-Bloch equation. We will show in the Section 4 that an Abelian loop group $L T$, where $T$ is a certain maximal torus in $G$, acts in a Hamiltonian way on our system. The corresponding conserved quantities will be constructed as the components of the moment map associated to the action of $L T$. Thus, we shall obtain a new family of conserved quantities.

The rewriting (2) of the Maxwell-Bloch equations appeared for the first time in the papers [9] and [10] by Q-Han Park and H.J. Shin. Without previous knowledge of these papers, the author rediscovered this rewriting and used it in the paper [7]. In [9] and [10] the equation (2) is viewed as an equation of field theory, while in [7] it is treated as a continuous chain of C. Neumann oscillators. With respect to the generalized Maxwell-Bloch equation it is interesting to note the following. The authors of [10] show that certain cases of the generalized Maxwell-Bloch equation (2) with $S U(2)$ replaced, say, by $S U(3)$ or $S U(4)$ are actually physically meaningful. They describe various instances of degenerate and non-degenerate interaction of light with two and three level optical media. The correct choice of $\tau(x)$ is crucial here.

\section{Generalized Maxwell-Bloch equation}

First we will show that the equations (1) and (2) are indeed equivalent. Let us introduce the matrix valued functions $\rho(t, x): \mathbb{R} \times \mathbb{R} \rightarrow \mathfrak{s u}(2)$ and $F(t, x): \mathbb{R} \times \mathbb{R} \rightarrow \mathfrak{s u}(2)$ by the formulae

$$
\rho(t, x)=\left(\begin{array}{cc}
i D(t, x) & i P(t, x) \\
-i P(t, x) & -i D(t, x)
\end{array}\right), \quad F(t, x)=\frac{1}{2}\left(\begin{array}{cc}
i \beta & E(t, x) \\
-\bar{E}(t, x) & -i \beta
\end{array}\right) .
$$

In terms of $F$ and $\rho$ the Maxwell-Bloch equations become

$$
\rho_{t}=[\rho, F], \quad F_{t}+c F_{x}=[\rho, \sigma],
$$

where

$$
\sigma=\frac{1}{2}\left(\begin{array}{cc}
i & 0 \\
0 & -i
\end{array}\right)
$$

is the first Pauli matrix multiplied by $i$. The first of the above equations is a Lax equation. Its general solution is of the form

$$
\rho(t, x)=\operatorname{Ad}_{g(t, x)}(\tau(x)), \quad F(t, x)=-g_{t}(t, x) \cdot g^{-1}(t, x) .
$$

Here $g(t, x): \mathbb{R} \times \mathbb{R} \rightarrow S U(2)$ is a Lie group valued function and $\tau(x): \mathbb{R} \rightarrow \mathfrak{s u}(2)$ takes values in the Lie algebra $\mathfrak{s u}(2)$. If we insert (5) into the second equation of (4), we indeed get the second order equation

$$
\left(g_{t} g^{-1}\right)_{t}+c\left(g_{t} g^{-1}\right)_{x}=\left[\sigma, \operatorname{Ad}_{g}(\tau(x))\right] .
$$

We note that the diagonal terms of the matrix $F(t, x)$ are constant. Thus we see that MaxwellBloch equations are equivalent to the equation (6) together with the constraint

$$
\left\langle g_{t} g^{-1}, \sigma\right\rangle=\text { const }=-\beta .
$$


From the point of view of the original physical interpretation, it would be better to introduce the variables $\rho$ and $F$ in the formula (3) as Hermitian rather than skew-Hermitian matrices. This would be achieved by multiplying the matrices by $-i$. Then $\rho$ would indeed have the proper form of the projection on the quantum mechanical wave-function. We have adopted the skew-Hermitian form because it is more convenient from the mathematical point of view on the one hand, and because it is better suited to our mechanical interpretation of the Maxwell-Bloch equations on the other. Namely, being skew-Hermitian, the matrices $\rho$ and $F$ are the proper elements of the Lie algebra $\mathfrak{s u}(2)$ and this will facilitate our reasoning and calculations below.

From now on we shall denote the Killing form by $\langle-,-\rangle$. In the equation (6) we can replace the unknown function $g(t, x): \mathbb{R} \times \mathbb{R} \rightarrow S U(2)$ by the function $g(t, x): \mathbb{R} \times \mathbb{R} \rightarrow G$ which takes values in an arbitrary Lie group $G$, if only we replace $\sigma \in \mathfrak{s u}(2)$ and $\tau(x) \in \mathfrak{s u}(2)$ by an element $\sigma \in \mathfrak{g}$ and a curve $\tau(x): \mathbb{R} \rightarrow \mathfrak{g}$, where $\mathfrak{g}$ is the Lie algebra of $G$.

We can simplify the equation (6) to some extent. First we note the following. Let $g(t, x): \mathbb{R} \times$ $\mathbb{R} \rightarrow G$ be a solution of (6). Then it is easy to check that for every $h(x): \mathbb{R} \rightarrow G$ the function $f(t, x)=g(t, x) h(x): \mathbb{R} \times \mathbb{R} \rightarrow G$ is a solution of the equation

$$
\left(f_{t} f^{-1}\right)_{t}+c\left(f_{t} f^{-1}\right)_{x}=\left[\sigma, \operatorname{Ad}_{f}\left(h(x) \cdot \tau(x) \cdot h^{-1}(x)\right)\right] .
$$

Therefore, we can assume that $\tau(x): \mathbb{R} \rightarrow \mathfrak{t} \subset \mathfrak{g}$ is a map which takes values in a chosen maximal toroidal subalgebra $\mathfrak{t} \subset \mathfrak{g}$.

We obtain an important equation, if we perform the following reduction. Consider the equation (6) for the case when $G=S U(2)$, but let the unknown function $g(t, x)$ be constrained to take values only in a subgroup $U(1) \subset S U(2)$ :

$$
g(t, x)=\left(\begin{array}{cc}
\cos \phi(t, x) & \sin \phi(t, x) \\
-\sin \phi(t, x) & \cos \phi(t, x)
\end{array}\right): \mathbb{R} \times \mathbb{R} \rightarrow U(1) \subset S U(2) .
$$

Let $\sigma$ be the Pauli matrix $\sigma=\frac{1}{2} \operatorname{diag}(i,-i)$ and let $\tau(x) \equiv \sigma$. Then a calculation shows that the equation (6) gives

$$
\phi_{t t}+c \phi_{t x}=\sin \phi
$$

This equation is essentially the sine-Gordon equation.

\section{Hamiltonian structure}

Let us consider the spatially constant solutions $g(t): \mathbb{R} \rightarrow G$ of the equation (6). These solutions actually solve the equation

$$
\left(g_{t} g^{-1}\right)_{t}=\left[\sigma, \operatorname{Ad}_{g}(\tau)\right]
$$

The following proposition describes the Hamiltonian nature of the above ordinary differential equation.

Proposition 1. The equation (7) is the equation of motion of the system $\left(T^{*} G, \omega_{c}, H\right)$, where $\omega_{c}$ is the canonical symplectic form on the cotangent bundle $T^{*} G$ and the Hamiltonian function $H: T^{*} G \rightarrow \mathbb{R}$ is given by

$$
H\left(g, p_{g}\right)=\frac{1}{2}\left\|p_{g}\right\|^{2}+\left\langle\sigma, \operatorname{Ad}_{g}(\tau)\right\rangle .
$$


Proof. Let us trivialize the cotangent bundle $T^{*} G$ over the Lie group $G$ by means of the right translations, $T^{*} G \cong G \times \mathfrak{g}$. With this trivialization in mind we shall denote the elements in the tangent spaces $T_{\left(g, p_{g}\right)}\left(T^{*} G\right) \cong \mathfrak{g} \times \mathfrak{g}^{*}$ by $\left(X_{b}, X_{c t}\right)$. The canonical symplectic form $\omega_{c}$ on the cotangent bundle $T^{*} G$ over the Lie group $G$ can be given by the formula

$$
\left(\omega_{c}\right)_{\left(g, p_{g}\right)}\left(\left(X_{b}, X_{c t}\right),\left(Y_{b}, Y_{c t}\right)\right)=-\left\langle X_{c t}, Y_{b}\right\rangle+\left\langle Y_{c t}, X_{b}\right\rangle+\left\langle p_{g},\left[X_{b}, Y_{b}\right]\right\rangle .
$$

Above $\langle-,-\rangle$ denotes the pairing between the elements of $\mathfrak{g}$ and those of $\mathfrak{g}^{*}$. For the proof of this formula see [11].

A solution of a Hamiltonian system is an integral curve of the Hamiltonian vector field $X_{H}$ and thus is given by the relation $\mathrm{d} H=\omega\left(X_{H},-\right)$, where $\omega$ is the symplectic form. For the Hamiltonian given by (8) we have

$$
\left\langle\mathrm{d} H,\left(\delta_{b}, \delta_{c t}\right)\right\rangle=-\left\langle\left[\sigma, \operatorname{Ad}_{g}(\tau)\right]^{b}, \delta_{b}\right\rangle+\left\langle\delta_{c t}, p_{g}^{\sharp}\right\rangle,
$$

where $b: \mathfrak{g} \rightarrow \mathfrak{g}^{*}$ and $\sharp: \mathfrak{g}^{*} \rightarrow \mathfrak{g}$ are defined by $\alpha^{b}=\langle\alpha,-\rangle$ and $\beta=\left\langle\beta^{\sharp},-\right\rangle$. Let us denote $X_{H}=\left(X_{b}, X_{c t}\right)$. Then we have

$$
\begin{aligned}
\left(\omega_{c}\right)_{\left(g, p_{g}\right)}\left(\left(X_{b}, X_{c t}\right),\left(\delta_{b}, \delta_{c t}\right)\right) & =-\left\langle X_{c t}, \delta_{b}\right\rangle+\left\langle\delta_{c t}, X_{b}\right\rangle+\left\langle p_{g},\left[X_{b}, \delta_{b}\right]\right\rangle \\
& =\left\langle-X_{c t}-\left\{X_{b}, p_{g}\right\}, \delta_{b}\right\rangle+\left\langle\delta_{c t}, X_{b}\right\rangle,
\end{aligned}
$$

where $\{a, \alpha\}$ denotes the ad*-action of $a \in \mathfrak{g}$ on $\alpha \in \mathfrak{g}^{*}$. The above equations now yield

$$
p_{g}^{\sharp}=X_{b}, \quad\left[\sigma, \operatorname{Ad}_{g}(\tau)\right]^{b}=X_{c t}+\left\{X_{b}, p_{g}\right\},
$$

and therefore

$$
X_{b}=p_{g}^{\sharp}, \quad X_{c t}=\left[\sigma, \operatorname{Ad}_{g}(\tau)\right]^{b} .
$$

Let now $\gamma(t)=\left(g(t), p_{g}(t)\right): \mathbb{R} \rightarrow T^{*} G$ be a curve expressed in the right trivialization and let $\dot{\gamma}=\left(g_{t} g^{-1},\left(p_{g}\right)_{t}\right)$ be its tangent at the point $\left(g(t), p_{g}(t)\right)$. The above equations tell us that $\gamma(t)$ is an integral curve of the Hamiltonian vector field $X_{H}$, if and only if it satisfies the equation

$$
\left(g_{t} g^{-1}\right)_{t}=\left[\sigma, \operatorname{Ad}_{g}(\tau)\right] .
$$

In the case when $G=S U(2)$, the system $\left(T^{*} S U(2), \omega_{c}, H\right)$ is the C. Neumann system which describes the motion of a particle on $S^{3}=S U(2)$ under the influence of a quadratic potential. The quadratic form defining the potential has two double eigenvalues. To see this we only have to calculate explicitly the potential $\left\langle\sigma, \operatorname{Ad}_{g}(\tau)\right\rangle$ in the case when $g \in S U(2)$. The elements of $S U(2)$ are matrices of the form

$$
g=\left(\begin{array}{cc}
g_{1}+i g_{2} & g_{3}+i g_{4} \\
-g_{3}+i g_{4} & g_{1}-i g_{2}
\end{array}\right), \quad \operatorname{det}(g)=\sum_{i=1}^{4} g_{i}^{2}=1
$$

If we take

$$
\tau=\left(\begin{array}{cc}
i a & b+i c \\
-b+i c & -i a
\end{array}\right) \quad \text { and } \quad \sigma=\left(\begin{array}{cc}
i & 0 \\
0 & -i
\end{array}\right)
$$

we get

$$
\begin{aligned}
2\left\langle\sigma, \operatorname{Ad}_{g}(\tau)\right\rangle & =-\operatorname{Tr}\left(\sigma g \tau g^{-1}\right) \\
& =2 a\left(g_{1}^{2}+g_{2}^{2}-g_{3}^{2}-g_{4}^{2}\right)+4 b\left(-g_{1} g_{4}+g_{2} g_{3}\right)+4 c\left(g_{1} g_{3}+g_{2} g_{4}\right) .
\end{aligned}
$$


The matrix of the above quadratic form has indeed two double eigenvalues $\lambda=2\|\tau\|$ and $\mu=-\lambda$. Thus the system $\left(T^{*} G, \omega_{c}, H\right)$, where the Hamiltonian is given by (8), can be considered as a generalized C. Neumann oscillator. Its configuration space is the Lie group $G$ instead of the sphere.

The fact that the quadratic form of the potential of $\left(T^{*} S U(2), \omega_{c}, H\right)$ has two double eigenvalues means that the system has two circular symmetries. Moreover, the system $\left(T^{*} G, \omega_{c}, H\right)$, where $G$ is compact semi-simple, has generically two toroidal symmetries. Inspection of the Hamiltonian (8) immediately shows that our system is preserved under the right action $\rho_{t}^{r}(g)=$ $g \cdot t$ of the maximal torus $T_{\tau}=\exp _{\tau}$ and under the left action $\rho_{t}^{l}(g)=t \cdot g$ of the torus $T_{\sigma}=\exp \mathfrak{t}_{\sigma}$. Here $\mathfrak{t}_{\tau}$ and $\mathfrak{t}_{\sigma}$ denote the maximal toroidal subalgebras in $\mathfrak{g}$ which contain the elements $\tau$ and $\sigma$, respectively. We have taken into the account the fact that the canonical symplectic form $\omega_{c}$ on $T^{*} G$ is left and right-invariant. The symmetries $\rho^{r}$ and $\rho^{l}$ will enable us to construct in Section 4 the symmetries of the generalized Maxwell-Bloch equation.

Now we shall return to the partial differential equation (6). We shall concentrate on the spatially periodic case, which means that we shall stipulate

$$
g(t, x+2 \pi)=g(t, x), \quad \tau(x+2 \pi)=\tau(x) .
$$

In a somewhat more graphic way, the equation (6) can be written as

$$
\left(g_{t} g^{-1}\right)_{t}(t, x)=-\left.\frac{c}{\epsilon}\left(g_{t} g^{-1}(t, x-\epsilon)-g_{t} g^{-1}(t, x+\epsilon)\right)\right|_{\epsilon \rightarrow 0}+\left[\sigma, \operatorname{Ad}_{g(t, x)}(\tau(x))\right] .
$$

Consider the function $g\left(t, x_{0}\right)$ at a fixed value $x_{0}$ of the spatial coordinate. We can think of $g\left(t, x_{0}\right)$ as of the position of the generalized C. Neumann oscillator moving in $G$. Its acceleration depends on the potential $\left[\sigma, \operatorname{Ad}_{g}(\tau)\right]$ and on the velocities $\left(g_{t} g^{-1}\right)\left(t, x_{0} \pm \epsilon\right)$ of the neighbouring oscillators. It is thus natural to try to understand the equation (6) as the equation of motion for a continuous chain of the generalized C. Neumann oscillators. The oscillators interact among themselves in a magnetic way. By this we mean that the acceleration of every given oscillator depends on the velocity and not on the position of its neighbours.

Let us now construct the Hamiltonian structure of the generalized Maxwell-Bloch equation which will correspond to the above interpretation. The obvious candidate for the configuration space of the continuous chain is the set of maps $g(x): S^{1} \rightarrow G$, or, in other words, the loop group $L G$. The phase space is therefore the cotangent bundle $T^{*} L G$. The natural choice for the Hamiltonian is the total energy of all the oscillators:

$$
H_{m b}\left(g(x), p_{g}(x)\right)=\int_{S^{1}}\left(\frac{1}{2}\left\|p_{g}(x)\right\|^{2}+\left\langle\sigma, \operatorname{Ad}_{g(x)}(\tau(x))\right\rangle\right) \mathrm{d} x .
$$

But the Hamiltonian system $\left(T^{*} L G, \omega_{c}, H_{m b}\right)$ does not correspond to the generalized MaxwellBloch equation (6). It is easily seen that the equation of motion of this system is simply the equation $\left(g_{t} g\right)_{t}(t, x)=\left[\sigma, \operatorname{Ad}_{g(t, x)}(\tau(x))\right]$ which describes the system of decoupled generalized C. Neumann oscillators. Therefore we have to modify this system in such a way that the magnetic-type interactions will be taken into account. We shall achieve this by perturbing the canonical symplectic form $\omega_{c}$ by an additional term.

Let $\Omega$ be the cocycle on the loop algebra $L \mathfrak{g}$ defining the central extension $\widetilde{L} \mathfrak{g}=L \mathfrak{g} \times \mathbb{R}$. Recall that $\Omega$ is given by

$$
\Omega(\xi(x), \eta(x))=-\int_{S^{1}}\left\langle\xi^{\prime}(x), \eta(x)\right\rangle, \quad \xi(x), \eta(x) \in L \mathfrak{g} .
$$

Let us denote by $\widetilde{\omega}_{m}$ the right-invariant 2 -form on the loop group $L G$ whose value at the identity is equal to $\Omega$, that is $\left(\widetilde{\omega}_{m}\right)_{e}=\Omega$. Finally let $\pi: T^{*} L G \rightarrow L G$ be the natural projection and let the 2 -form $\omega_{m}$ on $T^{*} L G$ be given as the pull-back $\omega_{m}=\pi^{*}\left(\widetilde{\omega}_{m}\right)$. We have the following theorem. 
Theorem 1. Let the Hamiltonian $H_{m b}$ in the Hamiltonian system $\left(T^{*} L G, \omega_{c}+c \omega_{m}, H_{m b}\right)$ be given by (12), and let $\omega_{m}$ be of the form described above. Then the equation of motion of this Hamiltonian system is the generalized Maxwell-Bloch equation (6).

Proof. The proof will be a modification of the proof of Proposition 1. Note first that the Killing form on $\mathfrak{g}$ induces an Ad-invariant inner product on $L \mathfrak{g}$ given by the formula

$$
\langle\langle\xi(x), \eta(x)\rangle\rangle=\int_{S^{1}}\langle\xi(x), \eta(x)\rangle \mathrm{d} x .
$$

By the symbol $\langle\langle-,-\rangle\rangle$ we shall also denote the pairing between the elements of $L \mathfrak{g}$ and those of $L \mathfrak{g}^{*}$, as well as the induced inner product on the dual $L \mathfrak{g}^{*}$. With this notation the directional derivative of the Hamiltonian $H_{m b}$ in the direction $\left(\delta_{b}, \delta_{c t}\right)$ is given by

$$
\left\langle\left\langle\mathrm{d} H_{m b},\left(\delta_{b}, \delta_{c t}\right)\right\rangle\right\rangle=-\left\langle\left\langle\left[\sigma, \operatorname{Ad}_{g}(\tau)\right]^{b}, \delta_{b}\right\rangle\right\rangle+\left\langle\left\langle\delta_{c t}, p_{g}^{\sharp}\right\rangle\right\rangle,
$$

where the maps $\sharp$ and $b$ are defined in the same way as before.

The formula (9) from the Proposition 1 is valid for every Lie group, hence also for the loop group $L G$. Therefore we get the following expression for the form $\omega_{m}+c \omega_{m}$ :

$$
\begin{aligned}
& \left(\omega_{c}+c \omega_{m}\right)_{\left(g, p_{g}\right)}\left(\left(X_{b}, X_{c t}\right),\left(Y_{b}, Y_{c t}\right)\right) \\
& \quad=-\left\langle\left\langle X_{c t}, Y_{b}\right\rangle\right\rangle+\left\langle\left\langle Y_{c t}, X_{b}\right\rangle\right\rangle+\left\langle\left\langle p_{g},\left[X_{b}, Y_{b}\right]\right\rangle\right\rangle-c\left\langle\left\langle\left(X_{b}\right)_{x}, Y_{b}\right\rangle\right\rangle .
\end{aligned}
$$

The above two formulae now give us the following relation for the Hamiltonian vector field $X_{H_{m b}}=\left(X_{b}, X_{c t}\right)$ :

$$
\begin{aligned}
\left(\omega_{c}+\right. & \left.c \omega_{m}\right)_{\left(g, p_{g}\right)}\left(\left(X_{b}, X_{c t}\right),\left(\delta_{b}, \delta_{c t}\right)\right) \\
& =-\left\langle\left\langle X_{c t}, \delta_{b}\right\rangle\right\rangle+\left\langle\left\langle\delta_{c t}, X_{b}\right\rangle\right\rangle+\left\langle\left\langle p_{g},\left[X_{b}, \delta_{b}\right]\right\rangle\right\rangle-c\left\langle\left\langle\left(X_{b}\right)_{x}, \delta_{b}\right\rangle\right\rangle \\
& =\left\langle\left\langle-X_{c t}-c\left(X_{b}\right)_{x}^{b}-\left\{X_{b}, p_{g}\right\}, \delta_{b}\right\rangle\right\rangle+\left\langle\left\langle\delta_{c t}, X_{b}\right\rangle\right\rangle .
\end{aligned}
$$

Since the vectors $\delta_{b}$ and $\delta_{c t}$ are linearly independent, we get two equations for the components of the Hamiltonian vector field:

$$
X_{b}=p_{g}^{\sharp}, \quad X_{c t}+c\left(X_{b}\right)_{x}^{b}=\left[\sigma, \operatorname{Ad}_{g}(\tau)\right]^{b} .
$$

Let $\gamma(t, x)=\left(g(t, x), p_{g}(t, x)\right): \mathbb{R} \rightarrow T^{*} L G$ be an integral curve of the field $X_{H_{m b}}$ given in the right trivialization $T^{*} L G \cong L G \times L \mathfrak{g}$. Then we have $\dot{\gamma}=\left(g_{t} g^{-1},\left(p_{g}\right)_{t}\right)$ and thus from (13) finally

$$
\left(g_{t} g^{-1}\right)_{t}+c\left(g_{t} g^{-1}\right)_{x}=\left[\sigma, \operatorname{Ad}_{g}(\tau)\right] .
$$

\section{A family of conservation laws}

In this section we shall construct a family of conservation laws for the generalized Maxwell-Bloch system. This family will correspond to a certain group of symmetries of the Maxwell-Bloch system. The symmetries form a loop group. Hence the corresponding family of conservation laws will consist of infinitely many functionally independent elements.

Let, for the sake of simplicity, the loop $\tau(x)$ in the equation

$$
\left(g_{t} g^{-1}\right)_{t}+c\left(g_{t} g^{-1}\right)_{x}=\left[\sigma, \operatorname{Ad}_{g}(\tau)\right]
$$

be constant, $\tau(x) \equiv \tau$. Denote by $\mathfrak{t}_{\tau}$ the maximal toroidal subalgebra in $\mathfrak{g}$ which contains the element $\tau$. Let $T_{\tau}=\exp _{\tau}$ be the corresponding maximal torus in $G$. We notice immediately that our equation is invariant with respect to the right action

$$
\rho_{r(x)}(g(t, x))=g(t, x) \cdot r(x), \quad r(x): S^{1} \rightarrow T_{\tau}
$$


of the loop group $L T_{\tau} \subset L G$. Our conserved quantities will correspond to the $L T_{\tau}$-symmetry in the sense of Noether's theorem.

Equivalently, the generalized Maxwell-Bloch Hamiltonian system $\left(T^{*} L G, \omega_{c}+c \omega_{m}, H_{m b}\right)$ is invariant with respect to the canonical lifting $\varrho$ of the action $\rho$ onto the cotangent bundle $T^{*} L G$. It is easily checked that, in the trivialization of $T^{*} L G$ by the right translation, the action $\varrho: L T_{\tau} \times T^{*} L G \rightarrow T^{*} L G$ is given by

$$
\varrho_{r(x)}\left(g(x), p_{g}(x)\right)=\left(g(x) \cdot r(x), p_{g}(x)\right) .
$$

This is an action by symplectomorphisms. Indeed, the lifting to the cotangent bundle of an action on the base space is always symplectic. But this right action is symplectic also with respect to the form $\omega_{m}$, due to the fact that $\omega_{m}$ is right-invariant. Thus the action $\varrho$ is Hamiltonian, since $T^{*} L G$ is a simply connected space. Therefore this action has the moment map. Our conservation laws will be the components of the moment map corresponding to the action $\varrho$. We shall prove the following theorem.

Theorem 2. Let $\left(T^{*} L G, \omega_{c}+c \omega_{m}, H_{m b}\right)$ be the generalized Maxwell-Bloch system. Let $\xi(x) \in$ $L \mathfrak{t}_{\tau}$ be an arbitrary loop in $\mathfrak{t}_{\tau}$. Then the function $F_{\xi}: T^{*} L G \rightarrow \mathbb{R}$, which in the right trivialization of $T^{*} L G$ is defined by the formula

$$
F_{\xi}\left(g(x), p_{g}(x)\right)=\int_{S^{1}}\left(p_{g}\left(\operatorname{Ad}_{g}(\xi)\right)-\frac{c}{2}\left\langle g_{x} g^{-1}, \operatorname{Ad}_{g}(\xi)\right\rangle\right) \mathrm{d} x,
$$

is a first integral of this system. In other words, let

$$
g(t, x): \mathbb{R} \times S^{1} \rightarrow G
$$

be a solution of the Maxwell-Bloch equation (6). Then for every loop $\xi(x): S^{1} \rightarrow \mathfrak{t}_{\tau}$, the quantity

$$
G_{\xi}(g(t, x))=\int_{S^{1}}\left\langle g_{t} g^{-1}-\frac{c}{2} g_{x} g^{-1}, \operatorname{Ad}_{g}(\xi)\right\rangle \mathrm{d} x
$$

is constant with respect to time $t$.

Proof. The second part of the theorem follows immediately from the first part. Let $\gamma(t)=$ $\left(g(t), p_{g}(t)\right): \mathbb{R} \rightarrow T^{*} L G$ be a solution of the Hamiltonian system $\left(T^{*} L G, \omega_{c}+c \omega_{m}, H_{m b}\right)$, and let $g(t, x): \mathbb{R} \times S^{1} \rightarrow G$ be the map given by the curve $g(t)=\pi(\gamma(t)): \mathbb{R} \rightarrow L G$, where $\pi: T^{*} L G \rightarrow L G$ is the natural projection. Then

$$
g(t, x): \mathbb{R} \times S^{1} \rightarrow G
$$

is a solution of the Maxwell-Bloch equation (6). Again we shall use the trivialization $T^{*} L G \cong$ $L G \times \mathfrak{g}^{*}$ by the right translations. Denote by

$$
p r_{2}: T^{*} L G \cong L G \times \mathfrak{g}^{*} \rightarrow L \mathfrak{g}^{*}
$$

the projection on the second component. Then the Killing form on $G$ yields the identification

$$
\operatorname{pr}_{2}(\gamma(t))=p_{g}(t)=\left\langle g_{t}(t, x) g^{-1}(t, x),-\right\rangle .
$$

This clearly shows that

$$
F_{\xi}(\gamma(t))=\int_{S^{1}}\left\langle g_{t} g^{-1}-\frac{c}{2} g_{x} g^{-1}, \operatorname{Ad}_{g}(\xi)\right\rangle \mathrm{d} x=G_{\xi}(g(t, x))
$$

for every $t$. 
We shall now show that $F_{\xi}$ are integrals of the Maxwell-Bloch Hamiltonian system. We have already mentioned that our integrals are the components of the moment map corresponding to the action $\varrho$ of $T L_{\tau}$ on the Maxwell-Bloch system.

Let $\xi(x) \in L \mathfrak{t}_{\tau}$ be an arbitrary element in the Lie algebra $L \mathfrak{t}_{\tau}$. Denote by $\widetilde{\xi}$ the infinitesimal action of $\xi$ on $T^{*} L G$. By definition, the $\xi$-component $H_{\xi}$ of the moment map $\mu: T^{*} L G \rightarrow\left(L \mathfrak{t}_{\tau}\right)^{*}$ is given by the formula

$$
\mu\left(g, p_{g}\right)(\xi)=H_{\xi}\left(g, p_{g}\right)
$$

where $H_{\xi}: T^{*} L G \rightarrow \mathbb{R}$ is the function whose Hamiltonian vector field is the infinitesimal action $\widetilde{\xi}$. This means that

$$
\left(\mathrm{d} H_{\xi}\right)_{\left(g, p_{g}\right)}\left(\delta_{b}, \delta_{c t}\right)=\left(\omega_{c}+c \omega_{m}\right)\left(\widetilde{\xi},\left(\delta_{b}, \delta_{c t}\right)\right)=\omega_{c}\left(\widetilde{\xi},\left(\delta_{b}, \delta_{c t}\right)\right)+c \omega_{m}\left(\widetilde{\xi},\left(\delta_{b}, \delta_{c t}\right)\right)
$$

for every tangent vector $\left(\delta_{b}, \delta_{c t}\right) \in T_{\left(g, p_{g}\right)}\left(T^{*} L G\right)$. Let the functions $H_{\xi}^{c}, H_{\xi}^{m}: T^{*} L G \rightarrow \mathbb{R}$ be given by the formulae

$$
\left(\mathrm{d} H_{\xi}^{c}\right)_{\left(g, p_{g}\right)}\left(\delta_{b}, \delta_{c t}\right)=\left(\omega_{c}\right)_{\left(g, p_{g}\right)}\left(\widetilde{\xi},\left(\delta_{b}, \delta_{c t}\right)\right), \quad\left(\mathrm{d} H_{\xi}^{m}\right)_{\left(g, p_{g}\right)}\left(\delta_{b}, \delta_{c t}\right)=\left(\omega_{m}\right)_{\left(g, p_{g}\right)}\left(\widetilde{\xi},\left(\delta_{b}, \delta_{c t}\right)\right) .
$$

Then

$$
H_{\xi}=H_{\xi}^{c}+c H_{\xi}^{m}
$$

Here we ignore the non-relevant indeterminate additive constant, and we shall continue to do so below. For the canonical form we have $\omega_{c}=\mathrm{d} \theta$, where $\theta$ is the tautological 1 -form on $T^{*} L G$. Thus we have

$$
\left(\mathrm{d} H_{\xi}^{c}\right)_{\left(g, p_{g}\right)}\left(\delta_{b}, \delta_{c t}\right)=(\mathrm{d} \theta)_{\left(g, p_{g}\right)}\left(\widetilde{\xi},\left(\delta_{b}, \delta_{c t}\right)\right), \quad \text { therefore } \quad H_{\xi}\left(g, p_{g}\right)=\theta_{\left(g, p_{g}\right)}(\widetilde{\xi}) .
$$

The expression of the infinitesimal action in the right trivialization is given by

$$
\widetilde{\xi}\left(g, p_{g}\right)=\left.\frac{\mathrm{d}}{\mathrm{d} s}\right|_{s=0} \varrho_{h(s)}\left(g, p_{g}\right)=\left(\operatorname{Ad}_{g}(\xi), p_{g}\right) .
$$

Here $h(s):(-\epsilon, \epsilon) \rightarrow L T_{\tau}$ is a path, such that $h(0)=e$, and $\left.\frac{\mathrm{d}}{\mathrm{d} s}\right|_{s=0} h(s)=\xi \in L \mathfrak{t}_{\tau}$. From the definition of the tautological 1-form we now have

$$
H_{\xi}^{c}\left(g, p_{g}\right)=p_{g}(\widetilde{\xi})=\int_{S^{1}} p_{g}\left(\operatorname{Ad}_{g}(\xi)\right) \mathrm{d} x
$$

which gives the first summand in (14).

We claim the the magnetic component $H_{\xi}^{m}$ is given by the formula

$$
H_{\xi}^{m}\left(g, p_{g}\right)=-\frac{1}{2}\left\langle\left\langle g_{x} g^{-1}, \operatorname{Ad}_{g}(\xi)\right\rangle\right\rangle=-\frac{1}{2} \int_{S^{1}}\left\langle g_{x} g^{-1}, \operatorname{Ad}_{g}(\xi)\right\rangle \mathrm{d} x .
$$

We will calculate the derivative of $H_{\xi}^{m}$ at $\left(g, p_{g}\right)$ in the direction $\left(\delta_{b}, \delta_{c t}\right) \in T_{\left(g, p_{g}\right)}\left(T^{*} L G\right)$. Let $s \mapsto\left(g(s), p_{g}(s)\right)$ be a path, such that $\left(g(0), p_{g}(0)\right)=\left(g, p_{g}\right)$ and $\left.\frac{\mathrm{d}}{\mathrm{d} s}\right|_{s=0}\left(g(s), p_{g}(s)\right)=\left(\delta_{b}, \delta_{c t}\right)$ in the right trivialization. Derivation gives

$$
\begin{aligned}
\mathrm{d}\left(H_{\xi}^{m}\right)_{\left(g, p_{g}\right)}\left(\delta_{b}, \delta_{c t}\right) & =\left.\frac{\mathrm{d}}{\mathrm{d} s}\right|_{s=0} \int_{S^{1}}\left\langle g(s)_{x} g(s)^{-1}, \operatorname{Ad}_{g(s)}(\xi)\right\rangle \\
& =\int_{S^{1}}\left(\left\langle\left(\delta_{b}\right)_{x}, \operatorname{Ad}_{g}(\xi)\right\rangle+\left\langle g_{x} g^{-1},\left[\delta_{b}, \operatorname{Ad}_{g}(\xi)\right]\right\rangle\right) \mathrm{d} x
\end{aligned}
$$




$$
\begin{aligned}
& =\int_{S^{1}}\left(-\left\langle\delta_{b},\left(\operatorname{Ad}_{g}(\xi)\right)_{x}\right\rangle-\left\langle\delta_{b},\left[g_{x} g^{-1}, \operatorname{Ad}_{g}(\xi)\right]\right\rangle\right) \mathrm{d} x \\
& =-2 \int_{S^{1}}\left\langle\delta_{b},\left[g_{x} g^{-1}, \operatorname{Ad}_{g}(\xi)\right]\right\rangle \mathrm{d} x .
\end{aligned}
$$

On the other hand we have

$$
\left(\omega_{m}\right)_{\left(g, p_{g}\right)}\left(\widetilde{\xi},\left(\delta_{b}, \delta_{c t}\right)\right)=\int_{S^{1}}\left\langle\left(\operatorname{Ad}_{g}(\xi)\right)_{x}, \delta_{b}\right\rangle \mathrm{d} x=\int_{S^{1}}\left\langle\delta_{b},\left[g_{x} g^{-1}, \operatorname{Ad}_{g}(\xi)\right]\right\rangle \mathrm{d} x,
$$

that proves (15).

If we now put together the formulae (14), (15) and (16), we finally get

$$
H_{\xi}\left(g, p_{g}\right)=\int_{S^{1}}\left(p_{g}\left(\operatorname{Ad}_{g}(\xi)\right)-\frac{c}{2}\left\langle g_{x} g^{-1}, \operatorname{Ad}_{g}(\xi)\right\rangle\right) \mathrm{d} x
$$

that proves the theorem.

Our conservation laws can be expressed in a more compact and suggestive form. Let us choose an arbitrary point $x_{0} \in S^{1}$ and let $\varphi_{n}(x): S^{1} \rightarrow \mathbb{R}$ be a sequence of functions which approximates the Dirac delta function $\delta_{x_{0}}$ on $S^{1}$. For every function $f: S^{1} \rightarrow \mathbb{R}$ we then have

$$
\lim _{n \rightarrow \infty} \int_{S^{1}} f(x) \cdot \varphi_{n}(x) \mathrm{d} x=\int_{S^{1}} f(x) \cdot \delta_{x_{0}}(x) \mathrm{d} x=f\left(x_{0}\right) .
$$

Let $\hat{\xi} \in \mathfrak{t}_{\tau}$ be an arbitrary element and let $\xi_{n}(x) \in L \mathfrak{t}_{\tau}$ be elements in the loop algebra defined by $\xi_{n}(x)=\varphi_{n}(x) \cdot \hat{\xi}$. Then it follows from the above theorem that for every $n \in \mathbb{N}$ we have

$$
\int_{S^{1}}\left\langle g_{t} g^{-1}-\frac{c}{2} g_{x} g^{-1}, \operatorname{Ad}_{g}\left(\xi_{n}\right)\right\rangle \mathrm{d} x=\int_{S^{1}}\left(\left\langle g_{t} g^{-1}-\frac{c}{2} g_{x} g^{-1}, \operatorname{Ad}_{g}(\hat{\xi})\right\rangle(x) \cdot \varphi_{n}(x)\right) \mathrm{d} x=A_{n} .
$$

The sequence $\left\{A_{n}\right\}_{n \in \mathbb{N}}$ of constants is clearly convergent and its limit is

$$
\left\langle g_{t} g^{-1}-\frac{c}{2} g_{x} g^{-1}, \operatorname{Ad}_{g}(\hat{\xi})\right\rangle\left(x_{0}\right)=\int_{S^{1}}\left(\left\langle g_{t} g^{-1}-\frac{c}{2} g_{x} g^{-1}, \operatorname{Ad}_{g}(\hat{\xi})\right\rangle(x) \cdot \delta_{x_{0}}(x)\right) \mathrm{d} x .
$$

This proves the following corollary.

Corollary 1. For every element $\hat{\xi}$ in the maximal Abelian subalgebra $\mathfrak{t}_{\tau} \subset \mathfrak{g}$ and for every point $x_{0} \in S^{1}$, the quantity

$$
\chi_{x_{0}}(g(t, x))=\left\langle g_{t} g^{-1}-\frac{c}{2} g_{x} g^{-1}, \operatorname{Ad}_{g}(\hat{\xi})\right\rangle\left(x_{0}\right)
$$

is constant along every solution $g(t, x): \mathbb{R} \times S^{1} \rightarrow G$ of the generalized Maxwell-Bloch equation.

From the Ad-invariance of the Killing form $\langle-,-\rangle$ we see that for every $\hat{\xi} \in \mathfrak{t}_{\tau}$ the quantity

$$
\chi_{x_{0}}=\left\langle g^{-1} g_{t}-\frac{c}{2} g^{-1} g_{x}, \hat{\xi}\right\rangle\left(x_{0}\right)
$$

is constant. To put it more briefly, let $p_{\mathfrak{t}_{\tau}}: \mathfrak{g} \rightarrow \mathfrak{t}_{\tau}$ be the orthogonal projection. Then for every solution $g(t, x): \mathbb{R} \times S^{1} \rightarrow G$ and for every point $x_{0} \in S^{1}$ the projection

$$
p_{\mathfrak{t}_{\tau}}\left(\left(g^{-1} g_{t}-\frac{c}{2} g^{-1} g_{x}\right)\left(x_{0}\right)\right)
$$

is a constant element in $\mathfrak{t}_{\tau}$. 
Consider again the Hamiltonian function $H_{m b}\left(g, p_{g}\right)$ of the Maxwell-Bloch dynamical system $\left(T^{*} L G, \omega_{c}+c \omega_{m}, H_{m b}\right)$. We see that the left action $\rho_{s}^{l}(x)(g(x))=s(x) \cdot g(x)$ of the subgroup $L T_{\sigma} \subset L G$ preserves the Hamiltonian $H_{m b}$. Here $T_{\sigma}$ is the maximal torus in $G$ whose Lie algebra $\mathfrak{t}_{\sigma}$ contains the element $\sigma$. But the group $L T_{\sigma}$ does not preserve the magnetic part $\omega_{m}$ of the symplectic structure $\omega_{c}+c \omega_{m}$. Recall that the magnetic part is right invariant, but it is not left invariant - not even with respect to the action of $L T_{\sigma}$. It is easily seen that only the torus $T_{\sigma} \subset L T_{\sigma}$ (containing the constant loops) preserves the symplectic structure, and hence the whole Hamiltonian system. Calculations, similar to those above, only simpler, give us the proof of the following proposition.

Proposition 2. Let $\eta \in \mathfrak{t}_{\sigma}$ be an arbitrary element. Then for every solution $g(t, x): \mathbb{R} \times S^{1} \rightarrow G$ the quantity

$$
J_{\eta}(g(t, x))=\int_{S^{1}}\left\langle g_{t} g^{-1}, \eta\right\rangle \mathrm{d} x
$$

is a constant. This means that the element

$$
P_{\mathfrak{t}_{\sigma}}(g(t, x))=p_{\mathfrak{t}_{\sigma}}\left(\int_{S^{1}} g_{t} g^{-1} \mathrm{~d} x\right)
$$

of $\mathfrak{t}_{\sigma}$ is constant along every solution. Here $p_{\mathfrak{t}_{\sigma}}: \mathfrak{g} \rightarrow \mathfrak{t}_{\sigma}$ is again the orthogonal projection.

It is well known that the Maxwell-Bloch equations are integrable. This is also true for the generalized Maxwell-Bloch equations. In particular, they satisfy the zero-curvature condition

$$
V_{t}-U_{x}+[U, V]=0
$$

for the Lax pair

$$
U=-\left(-z \sigma+g_{t} g^{-1}\right) \quad \text { and } \quad V=-z \sigma+g_{t} g^{-1}-\frac{1}{z} \operatorname{Ad}_{g}(\tau) .
$$

The proof is a matter of trivial checking. We have to note that the conserved quantities constructed above do not comprise a complete system of first integrals. But they can be used to reduce the system. An interested reader can find various results concerning the integrability of the Maxwell-Bloch equations in the references [12, 13, 14, 15] etc.

As we mentioned above, the conserved quantities associated with the action of $L T_{\tau}$ can be used to construct symplectic reductions of the system $\left(T^{*} L G, \omega_{c}+c \omega_{m}, H_{m b}\right)$. One can see that the nature of the symplectic reductions $\mu^{-1}(\alpha) / L T_{\tau}$ depends quite heavily on the choice of the level $\alpha \in L \mathfrak{t}_{\tau}$ of the moment map. We intend to study some cases in another paper.

For the case of the original Maxwell-Bloch equation, these symplectic quotients can be thought of as infinite-dimensional analogues of the following situation. Let $\left(T^{*} S U(2), \omega_{c}, H\right)$,

$$
H\left(g, p_{g}\right)=\frac{1}{2}\left\|p_{g}\right\|^{2}+\left\langle\sigma, \operatorname{Ad}_{g}(\tau)\right\rangle,
$$

be the Hamiltonian system of the C. Neumann oscillator on $S^{3}$ with two circular symmetries, or equivalently, the Maxwell-Bloch system for the solutions which are constant with respect to the spatial variable. Then the right action of the maximal torus $T_{\tau}=\exp \mathfrak{t}_{\tau}$ on our system is Hamiltonian. Let $\mu: T^{*} G \rightarrow \mathfrak{t}_{\tau}$ be the corresponding moment map. The quotient $\left(\mu^{-1}(a) / T_{\tau}, \omega_{s q}, H_{s q}\right)$ is the system $\left(T^{*} S^{2}, \omega_{c}+a \omega_{m}, H_{s q}\right)$, where the induced Hamiltonian is given by

$$
H_{s q}\left(q, p_{q}\right)=\frac{1}{2}\left\|p_{q}\right\|^{2}+\langle\sigma, q\rangle
$$


and $q=\operatorname{Ad}_{g}(\tau) \in \mathcal{O}_{\tau}=S^{2} \subset \mathfrak{s u}(2)$. The magnetic perturbation $\omega_{m}$ of the symplectic form is given by

$$
\left(\widetilde{\omega}_{m}\right)_{\left(q, p_{q}\right)}\left(X_{1}, X_{2}\right)=p_{q}\left(\left[\kappa_{1}, \kappa_{2}\right]\right), \quad X_{i}=\left[\kappa_{i}, q\right] \in T_{q} \mathcal{O}_{\tau}=T_{q} S^{2} .
$$

The reduced system describes the charged spherical pendulum which moves in the magnetic field of a Dirac monopole situated in the centre of the sphere $S^{2}$. More details and the proof can be found for example in $[16,17]$. The quotient construction is easily generalized to the cases when $S U(2)$ is replaced by a compact semi-simple $G$. The sphere is then replaced by the general co-adjoint orbit $\mathcal{O}_{\tau}$ of $\tau$ and the magnetic form $\omega_{m}$ is just the well-known Kostant-Kirillov symplectic form which is a part of the Kähler structure on $\mathcal{O}_{\tau}$.

\section{Concluding remarks}

We have described a new Hamiltonian structure of the Maxwell-Bloch equations. The key step in the construction is the observation that the Maxwell-Bloch equations can be considered as the equation of motion of a continuous chain of C. Neumann oscillators on the three-dimensional sphere. These oscillators interact through forces of the magnetic type. Our Hamiltonian structure is derived from the well-known Hamiltonian structure of the C. Neumann oscillator. What has to be added in the case of the Maxwell-Bloch equations is the component which accounts for the magnetic-type interactions among the oscillators in the chain. This is achieved by means of the perturbation of the canonical symplectic structure by a topologically non-trivial magnetic term. This term is studied in more detail in [7]. Among other things, we show in [7] that in the Lagrangian formulation this term gives rise to essentially the same topologically non-trivial term as the one appearing in the Wess-Zumino-Witten theory. In this paper we have concentrated on finding a family of conservation laws of the Maxwell-Bloch equations. The task was facilitated exactly by the use of our particular Hamiltonian structure.

It is important to note that the Maxwell-Bloch equations might very well be endowed with additional Hamiltonian structures, different form the one described here. One such structure is given by Holm and Kovačič in [18]. It can be easily seen that the two structures are inequivalent. The symplectic structure presented in [18] does not include derivatives with respect to the spatial variable, while ours does. The existence of these two Hamiltonian structures suggests the possibility of the existence of a bi-Hamiltonian structure on the Maxwell-Bloch system. We intend to address this issue in another paper. Another interesting avenue to the study of Hamiltonian structures was suggested to the author by one of the referees. He/she points out the important fact that the reduced Maxwell-Bloch equations can re rewritten in the form of a nonlinear von Neumann equation. This is shown in [19]. A treatment of the nonlinear von Neumann-type equations and of their importance to quantum physics can be found in [20]. Some of the nonlinear von Neumann equations indeed have the Hamiltonian formulations of different sorts. An interesting example pointed out by the referee is the generalized Hamiltonian structure of Nambu, described in [21].

Let us briefly outline a somewhat more traditional way of describing the Hamiltonian structure of the reduced Maxwell-Bloch equations. The reduced system equations considered in [19] (and in other sources) is the system of three ordinary differential equations

$$
\begin{array}{ll}
\dot{u}_{1}=-\Delta u_{2}, & \Delta=\text { const }, \\
\dot{u}_{2}=\Delta u_{1}+k \mathcal{E} u_{3}, & k=\text { const }, \\
\dot{u}_{3}=-k \mathcal{E} u_{2}, & \mathcal{E}=(2 / k \tau) \operatorname{sech}(t / \tau) .
\end{array}
$$

Since the variables $u_{1}, u_{2}$ and $u_{3}$ are related by the constraint $u_{1}^{2}+u_{2}^{2}+u_{3}^{2}=1$, the phase space of this system is the two-dimensional sphere $S^{2}$. We can view $S^{2}$ as a co-adjoint orbit of 
a non-zero element in the Lie algebra $\mathfrak{s u}(2)$. If we introduce $\mathfrak{s u}(2)$-valued maps $\varrho: S^{2} \rightarrow \mathfrak{s u}(2)$ and $\Omega: S^{2} \times \mathbb{R} \rightarrow \mathfrak{s u}(2)$

$$
\varrho=\left(\begin{array}{cc}
i u_{3} & u_{1}+i u_{2} \\
-u_{1}+i u_{2} & -i u_{3}
\end{array}\right), \quad \Omega(t)=\frac{1}{2}\left(\begin{array}{cc}
i \Delta & \kappa \mathcal{E}(t) \\
-\kappa \mathcal{E}(t) & -i \Delta
\end{array}\right)
$$

then the system (17) can be written in the form

$$
\dot{\varrho}=[\Omega, \varrho] .
$$

Let us recall the Kostant-Kirillov symplectic form on the arbitrary co-adjoint orbit $\mathcal{O} \subset \mathfrak{g}$ given by the formula

$$
\left(\omega_{k k}\right)_{m}(X, Y)=m([a, b]), \quad X=[m, a], Y=[m, b] \in T_{m} \mathcal{O} .
$$

In the case of $\mathfrak{g}=\mathfrak{s u}(2)$, we can identify $\mathfrak{s u}(2)$ and the dual space $\mathfrak{s u}(2)^{*}$ via the Killing form $\langle\alpha, \beta\rangle=-\frac{1}{2} \operatorname{Tr}(\alpha \cdot \beta)$. Thus, on $S^{2} \subset \mathfrak{s u}(2)$ we get the expression

$$
\left(\omega_{k k}\right)_{\varrho}(X, Y)=\langle\varrho,[a, b]\rangle=-\frac{1}{2} \operatorname{Tr}(\varrho \cdot[a, b]), \quad X=[\varrho, a], Y=[\varrho, b] \in T_{\varrho} S^{2} .
$$

Let us now consider the function $H: S^{2} \times \mathbb{R} \rightarrow \mathbb{R}$ given by the formula

$$
H(\varrho, t)=\langle\varrho, \Omega(t)\rangle
$$

A short calculation shows that the reduced Maxwell-Bloch equations (18) are the equation of motion for the Hamiltonian system $\left(T^{*} S^{2}, \omega_{k k}, H\right)$ with the time-dependent Hamiltonian given by the formula (19).

We conclude this remark by observing that, in one way or another, the reduced MaxwellBloch equations lead to Hamiltonian structures which are different from the one considered in our paper. The main reason for this probably lies in the fact that in the case of the reduced equations, the electric field $\mathcal{E}$ is not an unknown, but it is assumed to be a known function of time, for example $\mathcal{E}(t)=(2 / k \tau) \operatorname{sech}(t / \tau)$.

In this paper, we studied the Maxwell-Bloch equations in their slowly varying envelopes approximation and in the sharp line limit, that is, without the inhomogeneous broadening. When the inhomogeneous broadening is taken into account, the resulting equations preserve the families of symmetries described in this paper. These symmetries can then be used for reduction and the resulting reduced equations would be analogous to those described above. However, the equations with broadening do not have a Hamiltonian structure directly analogous to the one described in this paper. For any Hamiltonian structure of the equations with broadening, the symplectic structure would have to be substantially different from ours. At this moment the author does not know, whether it would also carry over to the case without the assumption of the slowly varying envelopes.

\section{Acknowledgements}

I would like to thank professors Pavel Winternitz, Gregor Kovačič, and Jiř́ Patera for interesting and stimulating discussions. The research for this paper was supported in part by the research programme Analysis and Geometry P1-0291, Republic of Slovenia. A part of the research was done at the Centre de Recherches Mathématiques, Montreal, Canada. The hospitality of CRM and especially of professor Jiří Patera is gratefully acknowledged. 
[1] Reyman A.G., Semenov-Tian-Shansky M.A., Reduction of Hamiltonian systems, affine Lie algebras and Lax equations I, Invent. Math., 1979, V.54, 81-100.

[2] Reyman A.G., Semenov-Tian-Shansky M.A., Integrable systems II, in Encyclopaedia of Mathematical Sciences, Vol. 16, Editors V.I. Arnold and S.P. Novikov, Berlin, Springer, 1994, 116-259.

[3] Saksida P., Nahm's equations and generalizations of the Neumann system, Proc. Lond. Math. Soc., 1999, V.78, 701-720.

[4] Novikov S.P., The Hamiltonian formalism and multivalued analogue of Morse theory, Uspekhi Mat. Nauk, 1982, V.37, 3-49 (in Russian).

[5] Marsden J.E., Lectures on mechanics, London Mathematical Society Lecture Note Series, Vol.174, Cambridge, Cambridge University Press, 1992.

[6] Marsden J.E., Ratiu T.S., Introduction to mechanics and symmetry, New York, Springer, 1994.

[7] Saksida P., Maxwell-Bloch equations, C. Neumann systems and Kaluza-Klein theory, J. Phys. A: Math. Gen., 2005, V.38, 10321-10344.

[8] Kostant B., Quantization and unitary representations, in Lectures in Modern Analysis and Applications III. Lecture Notes in Math., Vol.179, Editor C.T. Taam, Berlin, Springer, 1970, 87-208.

[9] Park Q-Han, Shin H.J., Complex sine-Gordon equation in coherent optical pulse propagation, J. Korean Phys. Soc., 1997, V.30, 336-340, solv-int/9904007.

[10] Park Q-Han, Shin H.J., Field theory for coherent optical pulse propagation, Phys. Rev. A, 1998, V.57, 4621-4642, solv-int/9709002.

[11] Abraham R., Marsden J.E., Foundations of Mechanics, 2nd ed., Reading MA, Benjamin-Cummings, 1978.

[12] Lamb G.L., Phase variation in coherent-optical-pulse propagation, Phys. Rev. Lett., 1973, V.31, $196-199$.

[13] Lamb G.L., Coherent-optical-pulse propagation as an inverse problem, Phys. Rev. A, 1974, V.9, 422-430.

[14] Caudrey P.J., Eilbeck J.C., Gibbon J.D., An $N$-soliton solution of a nonlinear optics equation derived by inverse method, Lett. Nuovo Cimento, 1973, V.8, 773-779.

[15] Gabitov I.R., Zakharov V.E., Mikhailov A.V., The Maxwell-Bloch equation and the method of the inverse scattering problem, Teoret. Mat. Fiz., 1985, V.63, 11-31 (in Russian).

[16] Saksida P., Neumann system, spherical pendulum and magnetic fields, J. Phys. A: Math. Gen., 2002, V.35, 5237-5253.

[17] Saksida P., Integrable anharmonic oscillators on spheres and hyperbolic spaces, Nonlinearity, 2001, V.14, 977-994.

[18] Holm D., Kovačič G., Homoclinic chaos in a laser-matter system, Phys. D, 1992, V.56, 270-300.

[19] Naudts J., Kuna M., Special solutions of nonlinear von Neumann equations, math-ph/0506020.

[20] Czachor M., Kuna M., Leble S.B., Naudts J., Nonlinear von Neumann-type equations, in Trends in Quantum Mechanics (1998, Goslar), River Edge, NJ, World Sci. Publishing, 2000, 209-226, quant-ph/9904110.

[21] Nambu Y., Generalized Hamiltonian dynamics, Phys. Rev. D, 1973, V.7, 2405-2412. 